\title{
Influence of plasticity on magnetic and magnetostrictive behaviors of dual-phase steel
}

\author{
Said Lazreg \\ LMT-Cachan \\ ENS-Cachan/CNRS/UPMC/UniverSud Paris \\ 61 avenue du président Wilson \\ 94235-F Cachan cedex \\ Email: lazreg@1mt.ens-cachan.fr
}

\begin{abstract}
Magnetic and magnetostrictive behaviors of magnetic materials are very sensitive to mechanical stress and especially to plastic deformation. A model based on the decomposition of a plastified material into mechanically hard and soft phases has been proposed. An appropriate experimental procedure is presented in order to validate the model. The magnetic and magnetostrictive behaviors of a dual-phase steel are carried out. Measurements are made at unloaded stress and under various applied stress to observe a recovery phenomenon predicted by the modeling. Experiments and modeling are compared.
\end{abstract}

\section{INTRODUCTION}

The influence of plastic deformation on the magnetic behavior has been studied intensively in the past years. It is characterized by a strong non-linear degradation of the magnetic behavior associated to a shift of the magnetostrictive behavior [1], [2]; it can be interpreted in term of influence of internal stresses [1], that is the basement of a previous magneto-plastic model [3]. A new fast and simple modeling relevant for non destructive evaluation area is proposed in [4]. This model is based on the decomposition of the matrix of a plastified material into mechanically hard and soft phases leading to multiaxial residual stress fields. Once the residual stress is estimated, it is introduced as a loading of a multidomain model recently proposed [5]. We propose hereafter a panel of experiments in order to validate the modeling. Since the model is considering the material as a two phased material, a dual-phase steel has been chosen for the experiments. Measurements of plastified samples are made after tensile strengthening at unloaded stress and under various applied uniaxial stress to observe a recovery phenomenon predicted by the modeling. Experiments and modeling are compared.

\section{A BRIEF DESCRIPTION OF THE MODEL [4]}

The simplified tensile strengthening is considered (figure 1) leading to an axial plastic deformation $E^{p}$. The plastified material consists in two phases: a soft phase $s$ and a hard phase $h$, meaning that $s$ phase exhibits a lower yield stress and strengthening than the $h$ phase. $f_{s}$ and $f_{h}$ indicate the volume fraction of $s$ and $h$ phases. Considering on the other hand a macroscopic applied stress $\boldsymbol{\Sigma}$ and isotropic elasticity,

\author{
Olivier Hubert \\ LMT-Cachan \\ ENS-Cachan/CNRS/UPMC/UniverSud Paris \\ 61 avenue du président Wilson \\ 94235-F Cachan cedex \\ Email: hubert@1mt.ens-cachan.fr
}

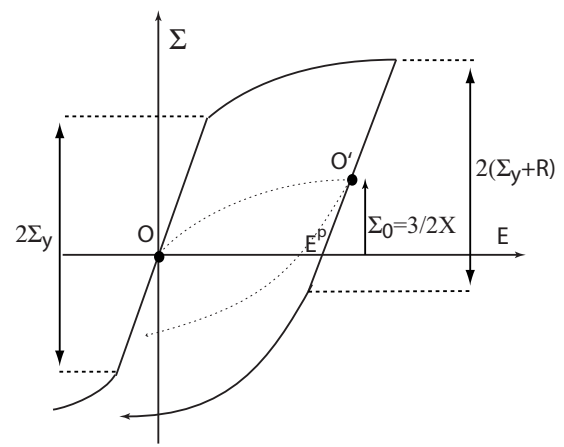

Fig. 1. Kinematic and isotropic variables identification in a classical stressstrain $\Sigma(E)$ diagram $-\Sigma_{y}$ : initial yield stress; $R$ : isotropic hardening. [6]

the stress tensors in $s$ and $h$ phases are:

$$
\boldsymbol{\sigma}_{s}=\boldsymbol{\Sigma}-\frac{3}{2} \mathbf{X} \quad \boldsymbol{\sigma}_{h}=\boldsymbol{\Sigma}+\frac{3}{2} \frac{f_{s}}{f_{h}} \mathbf{X}
$$

where $\mathbf{X}$ is the kinematic hardening component of the strengthening [6].

$\boldsymbol{\sigma}_{s}$ and $\boldsymbol{\sigma}_{h}$ are multiaxial tensors. Introduction of stress in the magneto-mechanical modeling requires to transform the tensors in scalars (equivalent stress). Following [7] and considering on the other hand a magnetic loading along the tensile axis, the magneto-mechanical equivalent stress in $s$ and $h$ phases is:

$$
\sigma_{s}^{e q}=\Sigma-\frac{9}{4} X \quad \sigma_{h}^{e q}=\Sigma+\frac{f_{s}}{f_{h}} \frac{9}{4} X
$$

$\Sigma$ is the macroscopic applied stress. $X$ is the component of $\mathbf{X}$ tensor along the loading axis. It can be experimentally identified following figure 1: the center of the yield surface is given by $\Sigma_{0}=\frac{3}{2} X$.

We can highlight two particular points:

1) At zero applied stress, the $s$ phase is submitted to compression, the $h$ phase to traction.

2) If a tensile stress $\Sigma=\frac{3}{2} \Sigma_{0}=\frac{9}{4} X$ is applied, the equivalent stress in the $s$ phase is null, leading to a possible recovery of the magnetic properties if the $h$ phase does not play any significant role. 
Magnetization $M$ and magnetostriction $\epsilon_{/ /}^{\mu}$ in the direction of the applied field of the $s$ and $h$ phases can be modeled separately thanks to the multidomain modeling [5], once the parameters of each phase (physical constants and adjusting parameters $A_{s}, \phi_{c}$ and $\theta_{c}$ ) are known. Considering on the other hand homogeneous magnetic field $H$, a mixing law allows the estimation of the magneto-mechanical behavior of the whole material:

$$
\begin{array}{r}
M(H, \Sigma)=f_{s} M_{s}\left(H, \sigma_{s}^{e q}\right)+f_{h} M_{h}\left(H, \sigma_{h}^{e q}\right) \\
\epsilon_{/ /}^{\mu}(H, \Sigma)=f_{s} \epsilon_{/ / s}^{\mu}\left(H, \sigma_{s}^{e q}\right)+f_{h} \epsilon_{/ / h}^{\mu}\left(H, \sigma_{h}^{e q}\right)
\end{array}
$$

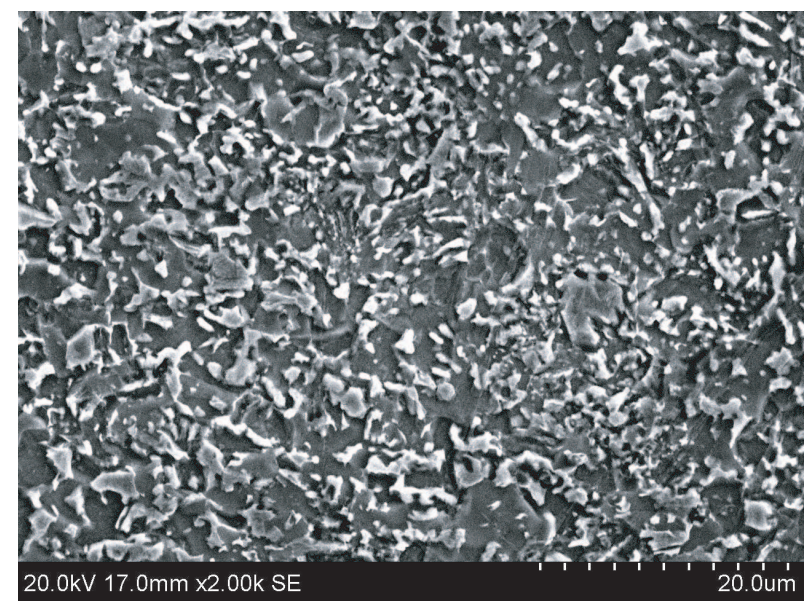

Fig. 2. Microstructure of the dual-phase steel - white: martensite islands; black: polycrystalline ferritic matrix.

\section{EXPERIMENTAL PROTOCOL}

A dual phase steel $(\mathrm{wt} \% \mathrm{C}=0.15$ ) is used for the study. Its microstructure consists of about $30 \%$ vol of hard martensite islands dispersed in a soft and ductile ferritic matrix (figure 2). As a first approximation, the martensite does not play any significant role in the magnetic behavior. The ferritic matrix can be considered as pure iron. The mixture of the two phases leads to a soft ferromagnetic material, that can be modeled by the multidomain modeling (considering $f_{s}=0.7$ ).

A MTS uniaxial electrohydraulic machine controlled by a computer (force or displacement) is used to carry out the stress-strain $\Sigma(E)$ behavior of the material. Unloading/reloading tests permit to estimate the kinematic $X$ and isotropic $R$ hardenings as function of the plastic strain $E^{p}$ thanks to a Cottrell's method (detection of non-linearity in compression - figure 1).

The experimental magnetic device enables in situ magnetic measurements on plastically deformed samples at unloaded state or under reloaded tensile stress (samples are $140 \mathrm{~mm}$ long $12.5 \mathrm{~mm}$ wide and $3 \mathrm{~mm}$ thick laminations) [8]. We used a primary winding to magnetize the samples, a $\mathrm{H}$-coil for the measurement of the magnetic field, a pick-up coil to measure the electromotive force, two shielded strain gauges (longitudinal and transverse directions - stuck on both faces) to estimate the plastic strain level and carry out the magnetostrictive behavior. Two ferrite yokes are put in contact with the sample to close the magnetic circuit and reduce the demagnetising field. Measurements are first performed on unstrained samples leading to the reference magnetic and magnetostrictive state. Measurements are next performed on samples submitted to static uniaxial stress below the yield stress to provide an evaluation of the magneto-elastic effect. Once exceeding the yield stress and for a given plastic deformation level $E^{p}$, magnetic measurements are performed at unloaded state $(\Sigma=0)$ and under increasing reloaded stress $\Sigma$ below the new yield stress. We must take care that the unloading always remains elastic.

Magnetic measurements reported here are the anhysteretic magnetic behavior $M(H)$ and the longitudinal anhysteretic magnetostrictive behavior $\epsilon_{/ /}^{\mu}(M)$. The anhysteretic curves are measured point by point by applying a sinusoidal magnetic field of mean value $H$, and of exponentially decreasing amplitude [8]. Three plastic deformation levels have been investigated: $0.1 \%, 1 \%$ et $3 \%$.

\section{EXPERIMENTAL RESULTS}

\section{A. Mechanical behavior}

Figure 3 reports the tensile stress-plastic strain behavior of the material. A high level of kinematic hardening $X$ is observed in accordance with the strong heterogeneity of the material. Isotropic hardening $R$ is on the contrary negligible.

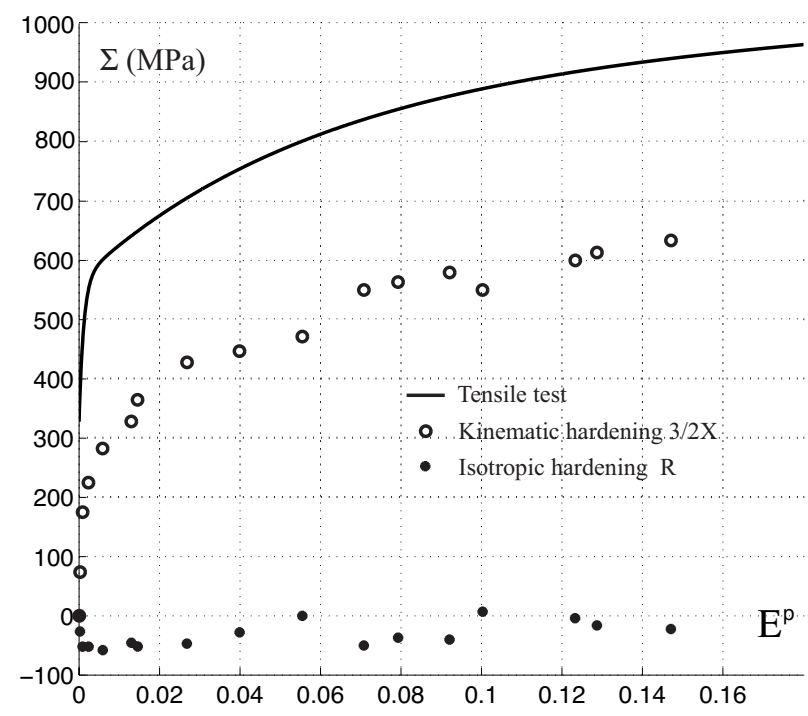

Fig. 3. Stress - plastic strain behavior of dual-phase steel - Associated isotropic and kinematic hardenings.

\section{B. Effect of plastic straining: unloaded state}

Figure 4 shows the effect of the three plastic strain levels on the magnetic behavior. A strong non linear degradation is observed as expected. Figure 5 reports the associated magnetostrictive behavior. The plastic strain acts clearly as 
a compressive stress effect as foreseen by the theoretical approach.

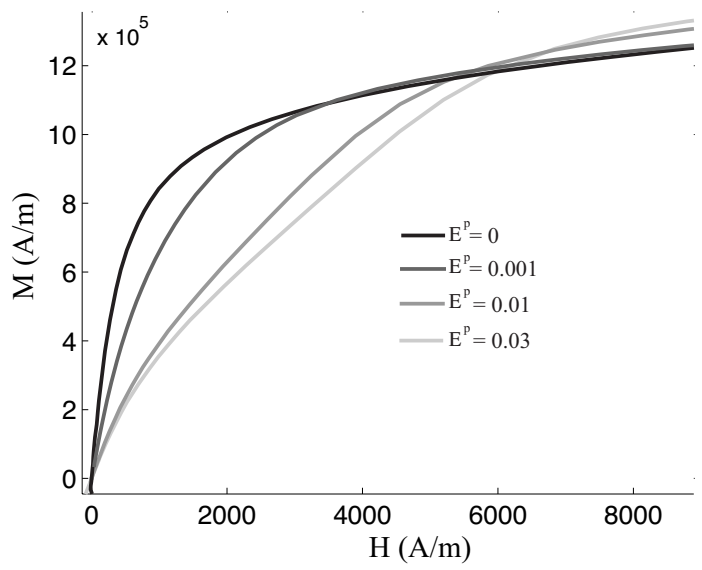

Fig. 4. Anhysteretic magnetic behavior of plastic strained samples at unloaded state

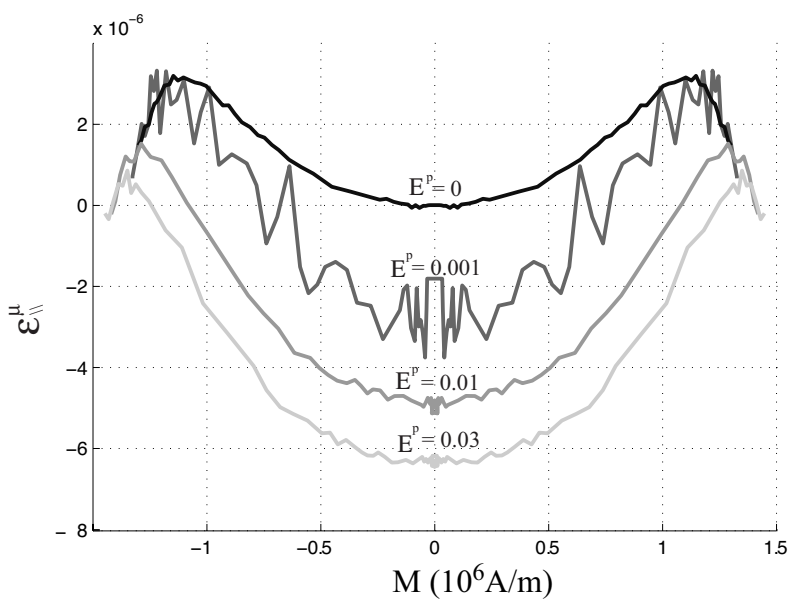

Fig. 5. Longitudinal anhysteretic magnetostrictive behavior of plastic strained samples at unloaded state

\section{Effect of plastic straining: reloaded state}

The prestrained samples are submitted to an increasing level of tensile stress. Magnetic and magnetostrictive measurements are performed. Figure 6 shows the evolution of the magnetic behavior of the sample prestrained at $3 \%$ and reloaded at various stress levels indicated in the figure. We observe that a critical stress $\Sigma_{c}^{\chi}$ allows to recover the initial magnetic behavior of the material. This result was expected since only the soft phase is supposed to participate to the magnetic behavior of the material. The tensile stress level $\frac{9}{4} X$ must have been reached following the theoretical approach. Figure 7 plots the initial susceptibility $\chi_{0}$ of the non-deformed and three deformed samples as function of the reloaded stress. $\chi_{0}$ appears to be entirely recovered reaching $2.10^{3}$ for three different applied stress $\Sigma_{c}^{\chi}$.

Figure 8 shows the longitudinal magnetostrictive behavior of the $3 \%$ plastically deformed specimen reloaded at various

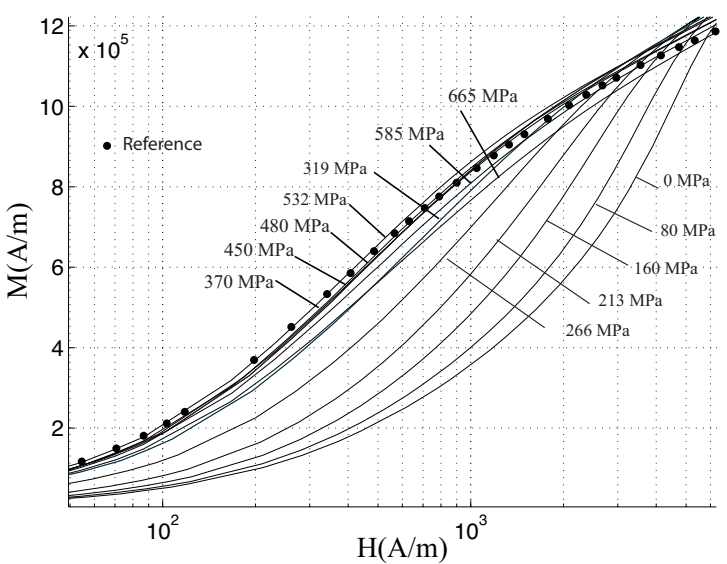

Fig. 6. Anhysteretic magnetic behavior of the sample prestrained at $3 \%$ and reloaded at various stress levels.

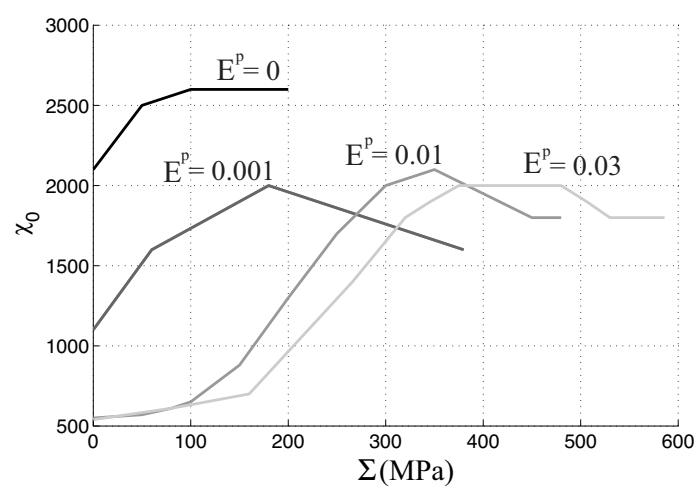

Fig. 7. Initial susceptibility of the samples as function of the reloaded stress.

stress levels indicated in the figure. As for the magnetic behavior, a critical stress allows to recover the initial magnetostrictive behavior of the material. It is denoted $\Sigma_{c}^{\chi}$. Below $\Sigma_{c}^{\chi}$, the material looks like an unstrained material submitted to a compression. Above $\Sigma_{c}^{\mu}$, the material looks like an unstrained material submitted to tensile stress. The phenomenon is reaching a saturation stage at higher level of applied stress. The so-called $\Delta E$ effect $\left(E_{/ /}^{\mu}(M=0, \Sigma)\right.$ behavior) has been plotted in figure 9 for all specimens in order to quantify the effect of the reloaded stress and accurately estimate $\Sigma_{c}^{\mu}$. $\Sigma_{c}^{\mu}$ is corresponding to the stress value at the intersection of $E_{/ /}^{\mu}(M=0, \Sigma)$ curves with $E_{/ /}^{\mu}=0$ axis.

\begin{tabular}{|l|c|c|c|}
\hline$E^{p}$ & $\Sigma_{c}^{\chi}(\mathrm{MPa})$ & $\Sigma_{c}^{\mu}(\mathrm{MPa})$ & $\frac{3}{2} \Sigma_{0}(\mathrm{MPa})$ \\
\hline \hline 0.001 & 180 & 80 & 225 \\
\hline 0.01 & 300 & 260 & 405 \\
\hline 0.03 & 380 & 340 & 540 \\
\hline
\end{tabular}

TABLE I

RECOVERY STRESSES - EXPERIMENTALY ESTIMATED $\left(\Sigma_{c}^{\chi}, \Sigma_{c}^{\mu}\right)$ AND FORESEEN BY THE MODEL $\left(\frac{3}{2} \Sigma_{0}\right)$.

Table I includes the recovery stresses as experimentally estimated $\left(\Sigma_{c}^{\chi}, \Sigma_{c}^{\mu}\right)$ and predicted by the model $\left(\frac{3}{2} \Sigma_{0}\right)$. Values 


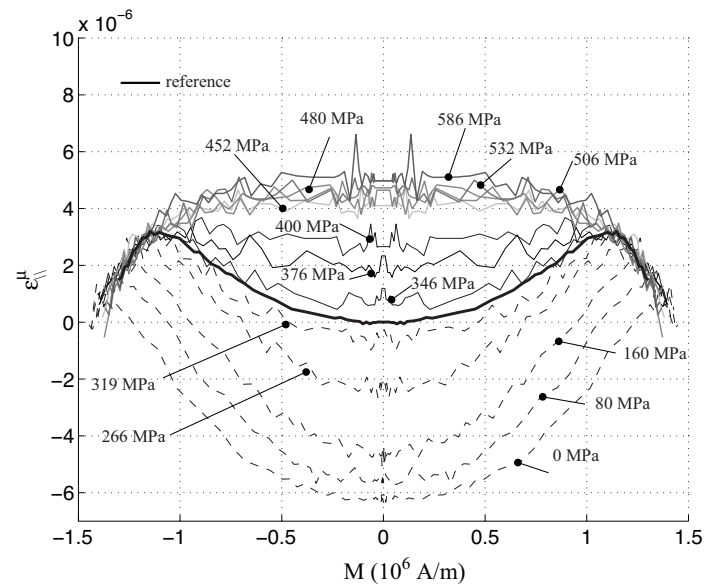

Fig. 8. Anhysteretic longitudinal magnetostrictive behavior of the sample prestrained at $3 \%$ and reloaded at various stress levels.

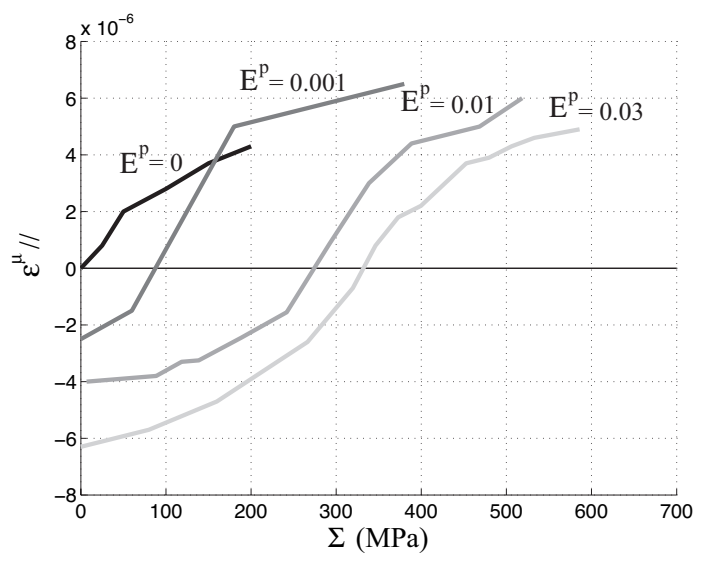

Fig. 9. Illustration of $\Delta E$ effect of the plastified samples.

of $\Sigma_{c}^{\chi}$ and $\Sigma_{c}^{\mu}$ are in accordance. The recovery stress predicted by the model is excessive.

\section{COMPARISON BETWEEN EXPERIMENTS AND MODELING}

The multidomain modeling is applied. The martensite is not taken in account. The physical constants used for the modeling (see [4]) correspond to pure iron $\left(M_{s}=1.71 \times 10^{6} \mathrm{~A} / \mathrm{m}\right.$; $\left.K_{1}=42.5 \times 10^{3} \mathrm{~J} . \mathrm{m}^{-3} ; \lambda_{100}=21 \times 10^{-6} ; \lambda_{111}=-21 \times 10^{-6}\right)$. Parameters $A_{s}, \phi_{c}$ and $\theta_{c}$ are optimized: $A_{s}=1 \times 10^{-3} \mathbf{J}^{-1} \cdot \mathrm{m}^{3}$; $\phi_{c}=40^{\circ} ; \theta_{c}=80^{\circ}$. The results of the magneto-mechanical modeling are plotted in figures 10 and 11 for the magnetization and longitudinal magnetostrictive behaviors. Results have to be compared to figures 4 and 5 . We observe a good adequacy between experimental results and modeling. The sensivity of the model to plastic strain seems nevertheless too high and saturation appears to be reached too quickly.

\section{CONCLUSION}

The experimental procedure presented in this study allows a qualitative validation of a multidomain modeling applied to the plastic straining thanks to a composite model. The role of martensite in the magnetic behavior should be taken

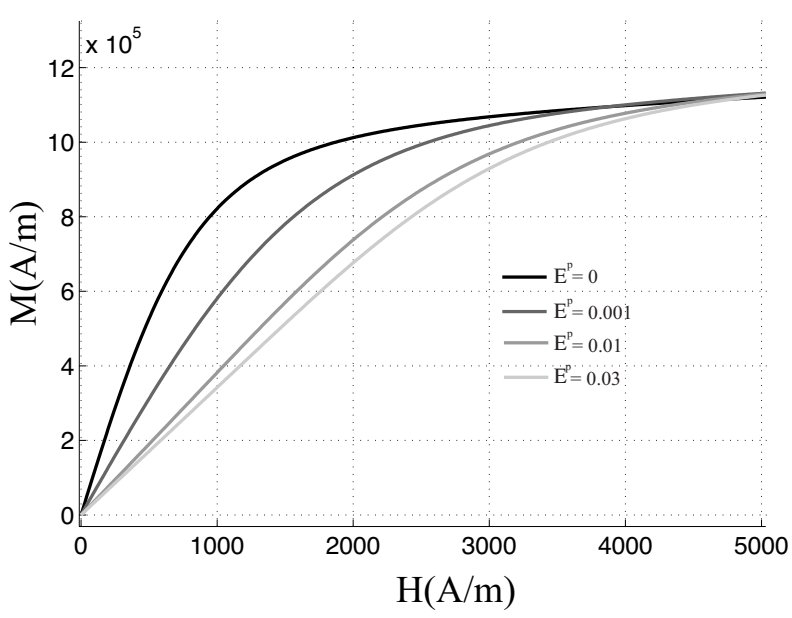

Fig. 10. Results of the modeling of the magnetic behavior after plastic deformation.

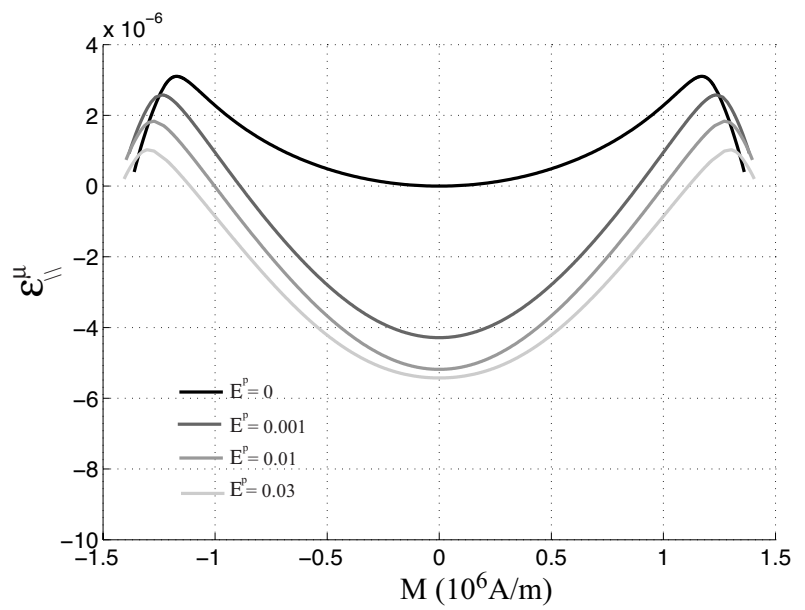

Fig. 11. Results of the modeling of the magnetostrictive behavior after plastic deformation.

in account for a better result. Nevertheless this model is not able to predict the influence of plastic deformation on coercive field or hysteresis losses since the pinning effect due to the metallurgical defects is not considered. These results and additional comments will be available in a paper being written. Magnetic measurement appears finally as an interesting way to evaluate the kinematic hardening in magnetic materials.

\section{REFERENCES}

[1] E. Hug, et al., Mat. Sc. and Eng., A332, (2002) p.193.

[2] O. Hubert, L. Daniel, J. of Magn. Magn .Mater., 304, (2006) p.e489.

[3] O. Hubert, et al., Steel Res. Int., 6, (2005), p.440.

[4] O. Hubert, S. Lazreg, Multidomain modeling of the influence of plastic deformation on the magnetic behavior for NDE application, SMM20, (2011).

[5] S. Lazreg, O. Hubert, J. Appl. Phys., 109, (2011), 07E508.

[6] J. Lemaitre, J.L. Chaboche Mechanics of solid materials, Cambridge University Press, 1994

[7] O. Hubert, L. Daniel, J. of Magn. Magn. Mater., 323, (2011), p.1766.

[8] O. Hubert et al., J. Magn. Magn. Mater., 254:255C, (2003), p.352. 